MATHEMATICS OF COMPUTATION

Volume 72 , Number 241, Pages $17-40$

S 0025-5718(01)01378-3

Article electronically published on July 13, 2001

\title{
LAVRENTIEV REGULARIZATION + RITZ APPROXIMATION $=$ UNIFORM FINITE ELEMENT ERROR ESTIMATES FOR DIFFERENTIAL EQUATIONS WITH ROUGH COEFFICIENTS
}

\author{
ANDREW KNYAZEV AND OLOF WIDLUND
}

\begin{abstract}
We consider a parametric family of boundary value problems for a diffusion equation with a diffusion coefficient equal to a small constant in a subdomain. Such problems are not uniformly well-posed when the constant gets small. However, in a series of papers, Bakhvalov and Knyazev have suggested a natural splitting of the problem into two well-posed problems. Using this idea, we prove a uniform finite element error estimate for our model problem in the standard parameter-independent Sobolev norm. We also study uniform regularity of the transmission problem, needed for approximation. A traditional finite element method with only one additional assumption, namely, that the boundary of the subdomain with the small coefficient does not cut any finite element, is considered.

One interpretation of our main theorem is in terms of regularization. Our FEM problem can be viewed as resulting from a Lavrentiev regularization and a Ritz-Galerkin approximation of a symmetric ill-posed problem. Our error estimate can then be used to find an optimal regularization parameter together with the optimal dimension of the approximation subspace.
\end{abstract}

\section{INTRODUCTION}

A particularly challenging class of problems arises with models described by partial differential equations (PDE's) with highly discontinuous coefficients. Many important physical problems are of this nature. In particular, they arise in the design and study of composite materials built from essentially different components; see, e.g., 9, 38, 7, 34, 24].

The fictitious domain/embedding method is another source of PDE's with highly discontinuous coefficients; cf., e.g., [39, 1, 12, 31]. In this method, the domain of the original boundary value problem is embedded into a larger one, where a new artificial boundary value problem is constructed. In the new, fictitious part of the domain the coefficients of PDE are chosen to be close to zero, if the original boundary condition is of Neumann type, or very large, in the Dirichlet case.

Received by the editor May 19, 1998 and, in revised form, December 28, 2000.

2000 Mathematics Subject Classification. Primary 65N30, 35R05; Secondary 35J25, 35J70.

Key words and phrases. Galerkin, Lavrentiev, Ritz, Tikhonov, discontinuous coefficients, error estimate, finite elements, regularization, regularity, transmission problem, fictitious domain, embedding.

The first author was supported by NSF Grant DMS-9501507.

The second author was supported in part by NSF Grant CCR-9732208 and in part by the U.S. Department of Energy under contract DE-FG02-92ER25127. 
There are several difficulties associated with the numerical solution of PDE's with a large jump in the coefficients as the problems are not uniformly well-posed with respect to the jump. The most serious difficulty is that an approximation, e.g., by a finite element method (FEM), of the PDE's may be very inaccurate. There are two main reasons for that: the lack of smoothness of the solution and the jump in the coefficients.

It is well known that solutions of problems with rough coefficients are generally not smooth, and then the usual finite element method cannot provide an accurate approximation; see, e.g., [20]. One way of approximating a nonsmooth function involves extra, special trial functions, to augment the standard FEM space, in order to approximate the nonsmooth part of the function. Finding such trial functions will usually require some knowledge of the form of the singularity, which is not always available. Another possibility is to employ refined meshes in the areas of low regularity; these areas are often known in advance.

In this paper, however, the lack of smoothness in the solution is not our primary concern. We shall assume that the solution can somehow be approximated by a trial subspace in a suitable Sobolev space. In our FEM example, we shall assume, for simplicity, that the coefficients are piecewise smooth and that the jumps in the coefficients appear across a smooth interface between subdomains.

Here we instead address a second difficulty, attributable to the large jump in the coefficients, concentrating on a case of a small coefficient in a subdomain, which results in a problem which is not uniformly elliptic.

The standard approach of deriving a FEM error estimate for selfadjoint PDE problems is based on the fact that the FEM approximation is the best approximation in the energy norm. In our case of highly discontinuous coefficients, the energy norm is not uniformly equivalent (with respect to the jump) to a natural parameter-independent Sobolev norm. This standard approach will fail to give an error estimate in a nonweighted Sobolev space with a constant, independent of the jump. Therefore, energy norm based estimates are naturally considered for problems with highly discontinuous coefficients; there are also similar results based on a least squares technique (see [30]) in weighted Sobolev spaces. Energy norm based error estimates are perfectly adequate when the Sobolev norm of the solution goes to infinity with the jump, in other words, when the solution is not uniformly smooth. As a simple example, we can consider a homogeneous Dirichlet problem for a diffusion equation with a small diffusion coefficient in a subdomain and with a fixed, parameter-independent right-hand side. Then, not even the $H^{1}$ norm of the solution is uniformly bounded, and there is little hope to obtain uniform error estimates in that norm. However, the assumption that the right-hand side is independent of the jump is not always satisfactory from the physical point of view, e.g., it does not allow us to consider a cavity in a perforated domain as a limit case of a subdomain with a small, but positive, diffusion coefficient. If we instead assume that the right-hand side is also small in the subdomain, where the diffusion coefficient is small, then the solution is uniformly bounded in a related parameter-independent Sobolev norm, in this case, the $H^{1}$ norm; see [3] for the exact formulation of such an assumption. It is known (see, e.g., [29, 3]) that under such an assumption a problem with a cavity can indeed be treated as a limit of a problem with a vanishing coefficient in the corresponding subdomain. Equations determining homogenized properties of composite materials (see, e.g., 7], 34]) provide an example of practical problems where our assumption is satisfied. 
In such a case, the energy norm will clearly deteriorate in the subdomain where the coefficient is small, and energy norm-based error estimates do not provide much information on the accuracy in the subdomain. Our goal is to derive new error estimates, which would use the assumption that the amplitude of the right-hand side is consistent with the value of the coefficients in subdomains such that the solution is uniformly bounded in an appropriate parameter-independent Sobolev norm. We also want to take advantage of the assumption that the jump in the coefficients is "regular." While in our example the operator is not uniformly elliptic (with respect to the jump) and the problem is not uniformly well posed, we can nevertheless treat the problem as essentially well-posed because of our special assumptions on the discontinuity in coefficients.

Our approach does not cover truly degenerate elliptic equations (see, e.g., 28]) with arbitrary discontinuous coefficients and/or ultimate failure of the ellipticity condition. For such equations, the use of the energy norm, or a properly weighted Sobolev norm seems vital.

As an application of our abstract result, we derive a FEM error estimate for the diffusion equation with a large jump in coefficients between subdomains. For this application, we also study uniform regularity of the transmission problem, needed for approximation. We prove that the solution, under some natural assumptions, is somewhat smooth uniformly in the jump of coefficients.

In the present paper, we consider the FEM approximation only. However, we expect our technique might be helpful for other approximation schemes as well; see, e.g., [18, 16] 17].

Our main result, being stated for the case involving a small coefficient, can be trivially reformulated for the case of a large coefficient and provides a uniform error estimate in the absence of locking. Thus, it can be applied, e.g., to estimate the error of a conforming FEM for displacements for linear elasticity equations in the incompressible limit, provided that non-locking elements are used. Some problems with a large parameter that appear in penalty methods can also be treated using our approach.

The technical part of the paper starts with some simple, but relevant, theoretical results for a parametric family of abstract symmetric operator equations with a jump in the coefficients described by a small parameter; see Section 2 Such problems are not uniformly well-posed, in the traditional sense, when the constant gets small. However, they can be split naturally into two well-posed problems as suggested in 3, 4. Using the projection method for the original problem is equivalent to applying the projection method to these two problems separately.

By exploring this idea and analyzing the fine structure of the error, we prove, in Section 3] a new error estimate of the Ritz-Galerkin method applied to our operator equation. The estimate does not use the energy norm, and all the constants are explicitly displayed and shown to be independent of the parameter. Under the assumption that the amplitude of the corresponding right-hand side is small consistently with the parameter, our bound for the error is parameter independent.

It is interesting to note that our finite dimensional problem can be viewed as a result of a Lavrentiev regularization - an analog of the Tikhonov regularization for symmetric problems - for the Ritz-Galerkin approximation of an ill-posed problem with a null-space; see, e.g., the recent books [41], pp. 162-173, and [8], Chapter 4. Our error estimate can be used to find the optimal regularization parameter together with the optimal dimension of the approximation subspace. A similar 
result is known in the regularization theory (cf. [35, pp. 75-78) but is based on different assumptions and techniques. However, the question of the choice of the regularization parameter for a general symmetric ill-posed problem is beyond the scope of this paper.

In Section 4, we provide a simple example of our general theory. We apply our main result to estimate the error of the FEM approximation of a parametric family of boundary value problems for the diffusion equation where the diffusion coefficient equals a small constant in a subdomain. For simplicity, we only analyze a quite simple finite element method rather than one particularly constructed for "rough coefficient" problems; we consider piecewise linear finite elements and assume, in addition, that no element is cut by the boundary of the subdomain with the small coefficient. In such a setting our abstract estimate becomes a uniform FEM error estimate for the model problem in the standard parameter-independent Sobolev norm. Our theory can also provide an error estimate for the well-known case of a self-intersecting interface with a constant independent of the jump, but it will depend on the mesh size. Sufficient smoothness of the interface is important.

Finally, we recall that the numerical solution of the resulting algebraic system is also difficult as the matrix of the system is not uniformly well-posed with respect to the jump in coefficients. For a number of preconditioned iterative methods the larger the jumps of the coefficients, the slower the convergence. However, it has been shown in the continuous case (cf. [2, 3, 6]) that if a special initial guess is used, then the rate of convergence does not depend on the size of the jumps even if a standard preconditioned iterative method is used. A similar result has been established in [27, 4 for algebraic systems of linear equations with symmetric coefficient matrices. Such methods are closely related to the capacitance matrix methods; see, e.g., [36, 11. Many domain decomposition methods can also be used for the effective solution of problems with rough coefficients and can provide uniform convergence. A relationship of different methods of this sort has been discovered in 5 .

A preliminary version of this paper was published as a technical report UCDCCM 132, 1998, at the Center for Computational Mathematics, University of Colorado at Denver.

\section{An abstract equation}

In a real Hilbert space, we consider an abstract linear system $(A+\omega I) u=f$ with a bounded symmetric nonnegative definite operator $A$, and a positive parameter $\omega, 0<\omega \leq 1$, where $I$ is the identity operator. The condition number of the operator $A+\omega I$ may tend to infinity as $\omega$ tends to zero; our system may not be uniformly well posed. The operator $A$ may have a non-zero null-space $\operatorname{Ker}(A)$.

In the present paper, we call a linear subset, which is not necessarily closed, a subspace. If such a linear subset is closed, we call it a closed subspace. We note that the kernel of a bounded operator is always a closed subspace.

The subspace $\operatorname{Im}(A)$, the range of $A$, and its closure, $\overline{\operatorname{Im}(A)}$, which is also the orthogonal complement to the $\operatorname{kernel} \operatorname{Ker}(A)$, will play a key role in the present paper.

We first make a couple of simple, but important observations, based on results from [3, 4. 
Lemma 2.1. The subspace $\operatorname{Im}(A)$ and its closure $\overline{\operatorname{Im}(A)}$ are invariant with respect to the operator $A+\omega I$.

Lemma 2.2. Let

$$
f=f_{0}+f_{1}, f_{0} \in \operatorname{Ker}(A), f_{1} \in \overline{\mathbf{I m}(A)} .
$$

Then,

$$
u=u_{1}+\frac{1}{\omega} f_{0},
$$

where $u_{1} \in \overline{\mathbf{I m}(A)}$ is the solution of

$$
(A+\omega I) u_{1}=f_{1} .
$$

Lemma 2.3. Let us assume that

$$
A \geq c I \text { on } \operatorname{Im}(A)
$$

i.e.,

$$
(A v, v) \geq c(v, v), \forall v \in \mathbf{I m}(A) .
$$

Then, $\operatorname{Im}(A)=\overline{\mathbf{I m}(A)}$,

$$
A+\omega I \geq(c+\omega) I \text { on } \overline{\operatorname{Im}(A)},
$$

and the problem (2.1) is well-posed on $\overline{\operatorname{Im}(A)}$ uniformly in $\omega, 0<\omega \leq 1$. This means that, for any given $f_{1} \in \overline{\operatorname{Im}(A)}$, there exists a unique solution $u_{1} \in \overline{\operatorname{Im}(A)}$ and that

$$
\left\|u_{1}\right\| \leq \frac{1}{c+\omega}\left\|f_{1}\right\|
$$

Informally speaking, under assumption (2.2) the problem $(A+\omega I) u=f$ is not truly ill-posed as it can be split into two problems which are uniformly well-posed in $\omega, 0<\omega \leq 1$.

Let us also mention that we do not use assumption (2.2) in our main Theorem 3.1. Instead, we shall assume a discrete analog of it, (3.4), in the next section.

\section{The Ritz-Galerkin Method FOR The ABstract EQUATION}

Let $P$ be an orthogonal projector on a closed trial subspace $\operatorname{Im}(P)$. We do not assume that $\operatorname{Im}(P)$ is necessarily finite dimensional, though in FEM applications this is usually the case.

Applying the Ritz-Galerkin method to the original equation $(A+\omega I) u=f$ gives

$$
P(A+\omega I) v=P f, v \in \mathbf{I m}(P) .
$$

Let $B=\left.P A\right|_{\operatorname{Im}(P)}$; then $0 \leq B=B^{*} \leq\|A\| I$. We can rewrite (3.1) as $(B+\omega I) v=$ $P f$ in $\operatorname{Im}(P)$.

We use the same approach as in the previous section when treating this equation. Let

$$
P f=(P f)^{0}+(P f)^{1},(P f)^{0} \in \mathbf{K e r}(B),(P f)^{1} \in \overline{\mathbf{I m}(B)} .
$$

Then,

$$
v=v^{1}+\frac{1}{\omega}(P f)^{0}
$$


where $v^{1}$ satisfies

$$
P(A+\omega I) v^{1}=(P f)^{1}, v^{1} \in \overline{\mathbf{I m}(B)} .
$$

The following lemma is an analog of Lemma 2.1.

Lemma 3.1. The subspace $\operatorname{Im}(B)$ and its closure $\overline{\operatorname{Im}(B)}$ are invariant with respect to the operator $B$ in $\operatorname{Im}(P)$ and to the operator $P(A+\omega I)$.

Lemma 3.2.

$$
\operatorname{Ker}(B)=\operatorname{Ker}(A) \cap \operatorname{Im}(P) .
$$

Proof. Clearly,

$$
\operatorname{Ker}(B) \supseteq \operatorname{Ker}(A) \cap \operatorname{Im}(P) .
$$

Let $u \in \mathbf{I m}(P)$, such that $P A u=0$. Then,

$$
0=(P A u, u)=(A u, u)=\left\|A^{1 / 2} u\right\|^{2} .
$$

Thus, $A^{1 / 2} u=0$ and $u \in \operatorname{Ker}(A)$.

Lemma 3.3. The vector $(P f)^{0} \in \operatorname{Ker}(A) \cap \mathbf{I m}(P)$ is the best approximation of the vector $f_{0}$ in the subspace $\operatorname{Ker}(A) \cap \mathbf{I m}(P)$, i.e.,

$$
\left(f_{0}-(P f)^{0}, v\right)=0, \forall v \in \mathbf{K e r}(A) \cap \mathbf{I m}(P) \text {. }
$$

Proof. Indeed,

$$
\left((P f)^{0}, v\right)=(P f, v)=(f, v)=\left(f_{0}, v\right)+\left(f_{1}, v\right)=\left(f_{0}, v\right)
$$

as $(P f)^{0} \in \operatorname{Ker}(A) \cap \operatorname{Im}(P)$ and $v \in \operatorname{Ker}(A) \cap \mathbf{I m}(P)$.

Lemma 3.4.

$$
\overline{\operatorname{Im}(B)}=\overline{P \operatorname{Im}(A)}
$$

Proof. We have

$$
\operatorname{Im}(B)=\operatorname{Im}(P A P) \subseteq \operatorname{Im}(P A)=P \operatorname{Im}(A),
$$

but this does not complete the argument.

Instead of the statement of the Lemma, we shall prove the following equivalent formula

Trivially,

$$
\operatorname{Ker}(P A P)=\operatorname{Ker}(A P)
$$

$$
\operatorname{Ker}(P A P) \supseteq \operatorname{Ker}(A P) .
$$

Now, let $P A P u=0$ for some vector $u$. Then

$$
0=(P A P u, u)=(A P u, P u)=\left\|A^{1 / 2} P u\right\|^{2} .
$$

Therefore, $A^{1 / 2} P u=0$ and $A P u=0$.

We have to make an important Assumption, analogous to (2.2),

$$
B \geq \tilde{c} I \text { on } \operatorname{Im}(B),
$$

i.e.,

$$
(B v, v) \geq \tilde{c}(v, v), \forall v \in \mathbf{I m}(B) .
$$

It is important to understand that assumption (3.4) always holds for finite dimensional approximations, i.e., when $\operatorname{dim} \operatorname{Im}(P)<\infty$. However, for essentially 
ill-posed problems the constant goes to zero when we make the subspace $\operatorname{Im}(P)$ larger in order to improve the approximation. In our later example, assumption (3.4) will be equivalent to a discrete extension theorem, Proposition 4.2, which is valid uniformly in the mesh size parameter, under some assumptions. Consequently, our example in the next section is not truly ill-conditioned.

Lemma 3.5. Under assumption (3.4), the subspace $\operatorname{Im}(B)$ is closed, $\operatorname{Im}(B)=$ $\overline{\operatorname{Im}(B)}$, and

$$
\operatorname{Im}(B)=P \overline{\mathbf{I m}(A)}
$$

Proof. The subspace $\operatorname{Im}(B)$ is closed by the Closed Graph Theorem of functional analysis. Then (3.5) follows from the previous lemma and the inclusion

$$
\operatorname{Im}(B) \subseteq P \operatorname{Im}(A)
$$

already given in Lemma 3.4

The following lemma can be established by using standard arguments.

Lemma 3.6. Under assumption (3.4), the following inequality holds

$$
P(A+\omega I)=B+\omega I \geq(\tilde{c}+\omega) I \text { on } \operatorname{Im}(B),
$$

and problem (3.2) is well-posed on $\operatorname{Im}(B)$ uniformly in $\omega$. The latter means that there exists an inverse operator $(B+\omega I)^{-1}: \operatorname{Im}(B) \rightarrow \operatorname{Im}(B)$ which is bounded uniformly in $\omega, 0<\omega \leq 1$, such that

$$
\left\|(B+\omega I)^{-1}\right\|_{\mathbf{I m}(B) \rightarrow \operatorname{Im}(B)} \leq \frac{1}{\tilde{c}+\omega}<\frac{1}{\tilde{c}},
$$

and for any given $f_{1} \in \overline{\mathbf{I m}(A)}$ there exists a unique solution $v^{1} \in \mathbf{I m}(B)$ such that

$$
\left\|v^{1}\right\| \leq \frac{1}{\tilde{c}+\omega}\left\|P f_{1}\right\| .
$$

Our goal is to estimate the norm of the difference of the exact solution $u$ of our original equation $(A+\omega I) u=f$ and its Ritz approximation $v$ given by (3.1):

$$
\|u-v\|=\left\|u_{1}-v^{1}+\frac{1}{\omega}\left\{f_{0}-(P f)^{0}\right\}\right\| .
$$

We first need some simpler estimates.

Lemma 3.7. The following estimate holds:

$$
\left\|P f_{1}-(P f)^{1}\right\| \leq\left\|f_{0}-(P f)^{0}\right\| .
$$

Proof. We have

$$
P f_{1}-(P f)^{1}=P f_{1}-\left\{P f-(P f)^{0}\right\}=(P f)^{0}-P f_{0}=P\left\{(P f)^{0}-f_{0}\right\} .
$$

Thus,

$$
\left\|P f_{1}-(P f)^{1}\right\|=\left\|P\left\{(P f)^{0}-f_{0}\right\}\right\| \leq\left\|f_{0}-(P f)^{0}\right\| .
$$

Lemma 3.8. Under assumption (3.4), let $w^{1} \in \mathbf{I m}(P)$ be the Ritz approximation of $u_{1} \in \overline{\operatorname{Im}(A)}$, the solution of (2.1), i.e.,

$$
P(A+\omega I) w^{1}=P f_{1} .
$$


Then, the following estimate holds:

$$
\left\|u_{1}-w^{1}\right\| \leq\left(1+\frac{\|(I-P) A P\|}{\tilde{c}+\omega}\right)\left\|u_{1}-P u_{1}\right\| .
$$

Proof. By the triangle inequality,

$$
\left\|u_{1}-w^{1}\right\| \leq\left\|u_{1}-P u_{1}\right\|+\left\|P u_{1}-w^{1}\right\| .
$$

We now estimate the second term.

We first notice that $P u_{1} \in \operatorname{Im}(B)$ and $P f_{1} \in \operatorname{Im}(B)$ by Lemma 3.5 as $u_{1} \in$ $\operatorname{Im}(A)$ and $f_{1} \in \operatorname{Im}(A)$. Then, $w^{1} \in \operatorname{Im}(B)$ by Lemmas 3.1 and 3.6 Thus, $P u_{1}-w^{1} \in \operatorname{Im}(B)$ and, using Lemma 3.6,

$$
\begin{aligned}
\left\|P u_{1}-w^{1}\right\| & =\left\|(B-\omega I)^{-1}(A-\omega I)\left(P u_{1}-w^{1}\right)\right\| \\
& \leq \frac{1}{\tilde{c}+\omega}\left\|(A-\omega I)\left(P u_{1}-w^{1}\right)\right\| \\
& =\frac{1}{\tilde{c}+\omega} \sup _{v \in \mathbf{I m}(B), v \neq 0} \frac{\left|\left((A+\omega I)\left(P u_{1}-w^{1}\right), v\right)\right|}{\|v\|} .
\end{aligned}
$$

By using the equality

$$
\left((A+\omega I)\left(u_{1}-w^{1}\right), v\right)=0, v \in \operatorname{Im}(P),
$$

which follows from $w^{1}$ being the Ritz approximation of $u_{1}$ in $\operatorname{Im}(P)$, we can estimate the numerator:

$$
\begin{aligned}
& \left|\left((A+\omega I)\left(P u_{1}-w^{1}\right), v\right)\right|=\left|\left((A+\omega I)\left(P u_{1}-u_{1}\right), v\right)\right| \\
& \quad=\left|\left(P A(I-P)\left(P u_{1}-u_{1}\right), v\right)\right| \leq\|(I-P) A P\|\left\|P u_{1}-u_{1}\right\|\|v\| .
\end{aligned}
$$

The statement of the lemma follows immediately.

Lemma 3.9. For $w^{1}$, defined in the previous lemma, we also have the estimate

$$
\left\|v^{1}-w^{1}\right\| \leq \frac{1}{\tilde{c}+\omega}\left\|P f_{1}-(P f)^{1}\right\|
$$

assuming that (3.4) holds.

Proof. Comparing equations (3.2) and (3.7), which define $v^{1}$ and $w^{1}$, shows that the operator is the same, but that the right-hand sides may differ,

$$
(B+\omega I)\left(v^{1}-w^{1}\right)=P f_{1}-(P f)^{1} .
$$

Now, by Lemma 3.5,

$$
P f_{1} \in P \overline{\mathbf{I m}(A)}=\mathbf{I m}(B) .
$$

Therefore, by Lemma 3.1, $w^{1} \in \operatorname{Im}(B)$ and so is $v^{1}$. Finally, Lemma 3.6 gives the estimate of the lemma.

Summarizing Lemmas 3.7 3.9, we have our main result.

Theorem 3.1. Under assumption (3.4), the following error estimate holds:

$$
\|u-v\| \leq C_{1} \operatorname{dist}\left\{u_{1} ; \mathbf{I m}(P)\right\}+C_{2} \operatorname{dist}\left\{\frac{1}{\omega} f_{0} ; \mathbf{I m}(P) \cap \operatorname{Ker}(A)\right\},
$$

where

$$
\begin{aligned}
& C_{1}=1+\frac{\|(I-P) A P\|}{\tilde{c}+\omega} \leq 1+\frac{\|(I-P) A P\|}{\tilde{c}}, \\
& C_{2}=\frac{\omega}{\tilde{c}+\omega}+1 \leq \frac{1}{\tilde{c}+1}+1<2,
\end{aligned}
$$


$u$ is the exact solution of $(A+\omega I) u=f$ and $v$ is its Ritz approximation given by (3.1).

Remark 3.1. Let us assume that we can approximate $u_{1}$ and $f_{0}$ accurately by properly choosing $P$ and that the constant $\tilde{c}$ does not tend to 0 . Then, the first term can be made small uniformly in $\omega, 0<\omega \leq 1$. If we make the natural assumption that the amplitude of $f_{0}$ is consistent with $\omega \rightarrow 0$, i.e., $f_{0}=O(\omega)$, which is equivalent to the assumption that the exact solution

$$
u=u_{1}+\frac{1}{\omega} f_{0}
$$

is uniformly bounded, see Lemmas $2.2,2.3$, then the second term in our theorem can also be made uniformly small. Thus, the theorem provides a $\omega$-uniform error estimate of the Ritz solution for a problem which is not formally $\omega$-uniformly wellposed.

Remark 3.2. The theorem can also be applied to a problem with a large parameter written in the following form

$$
\left(I+\frac{1}{\omega} A\right) u=g
$$

We simply take $f$ to satisfy $g=\omega f$ and use a scaling to return to the previous problem. If $g$ is parameter-independent, which is usually the case in practice, then the amplitude of $f_{0}$ is consistent with $\omega \rightarrow 0$ automatically, and we get a uniform estimate. Moreover, the amplitudes of $f_{1}$ and $u_{1}$ are then also consistent with $\omega \rightarrow 0$. This allows us to rewrite the estimate (3.10) in the following form:

$$
\|u-v\| \leq C_{3} \operatorname{dist}\left\{\frac{1}{\omega} u_{1} ; \mathbf{I m}(P)\right\}+C_{2} \operatorname{dist}\left\{\frac{1}{\omega} f_{0} ; \operatorname{Im}(P) \cap \operatorname{Ker}(A)\right\},
$$

where,

$$
C_{3}=\omega C_{1}=\omega+\|(I-P) A P\| \frac{\omega}{\tilde{c}+\omega} \leq 1+\|(I-P) A P\|, C_{2}<2 .
$$

This shows that assumption (B.4) is no longer needed. Let us note that in some practical applications the subspace $\operatorname{Im}(P) \cap \operatorname{Ker}(A)$ is too small, maybe even trivial, to provide a good approximation for $f_{0} \in \operatorname{Ker}(A)$. Such a situation is known as locking. Thus, our inequality (3.11) gives a uniform error estimate if there is no locking.

Remark 3.3. The term $\|(I-P) A P\|$ can be replaced by the simple upper bound $\|A\|$.

The following section gives an example of our general theory.

\section{EXAmple: FEM for the Diffusion EQUation}

To illustrate our results, we now consider a standard finite element method applied to the diffusion equation in two dimensions with a highly discontinuous diffusion coefficient:

$$
\operatorname{div}((k+\omega) \operatorname{grad} u-\phi)=0, u \stackrel{0}{1}(\square), \phi \in\left(L_{2}^{1}(\square)\right)^{2},
$$

where $\square$ is a polygonal simply connected domain. 
Let $\mathcal{D} \subset \square$ be a polygonal connected domain and let the open set $\mathcal{D}^{\perp}$ be defined by the conditions:

$$
\mathcal{D} \cap \mathcal{D}^{\perp}=\emptyset, \quad \overline{\mathcal{D}} \cup \overline{\mathcal{D}}^{\perp}=\square .
$$

We assume that $\mathcal{D}^{\perp}$ is a polygonal simply connected domain. To simplify the regularity results and extension theorems we need, let us also assume that $\mathcal{D}^{\perp}$ is strictly inside $\square$. This assumption, in particular, forces the intersection $\partial \mathcal{D} \cap \partial \square$ to have a positive Lebesgue measure on $\partial \square$,

$$
\operatorname{mes}\{\partial \mathcal{D} \cap \partial \square\}>0,
$$

which ensures that any function in $\stackrel{0}{W_{2}^{1}}(\square)$ that is constant in $\mathcal{D}$ vanishes there.

We assume that $0<\omega \leq 1$ and that $k$ is a piecewise constant function on $\square$, and highly discontinuous :

$$
k=0 \text { on } \mathcal{D}^{\perp}, k=1 \text { on } \mathcal{D} .
$$

To be able to approximate the solution, using a FEM subspace, we naturally need extra smoothness assumptions. We shall assume that the restrictions $\phi$ satisfy:

$$
\left.\phi\right|_{\mathcal{D}} \in\left(W_{2}^{\alpha}(\mathcal{D})\right)^{2},\left.\phi\right|_{\mathcal{D}^{\perp}} \in\left(W_{2}^{\alpha}\left(\mathcal{D}^{\perp}\right)\right)^{2}
$$

with a positive constant $\alpha<1 / 2$. For our first theorem of this section, Theorem 4.1 we shall also assume that

$$
\left.u\right|_{\mathcal{D}} \in W_{2}^{1+\alpha}(\mathcal{D})
$$

and we shall actually prove that this assumption holds uniformly in $\omega$ in Theorem 4.2 .

Because of the abstract nature of our main error estimate, it can be applied to any conforming FEM that fits inside the general Ritz-Galerkin framework. As an example, we consider the simplest FEM.

Let a standard finite element triangulation be constructed, with shape regular and quasi-uniform triangles, and denote the mesh size parameter by $h$. To prove a quasi-optimal error estimate, we need to assume, in addition, that none of the finite elements cuts the boundary $\partial \mathcal{D} \cup \partial \mathcal{D}^{\perp} \cup \partial \square$. Finally, we consider the FEM subspace of $\stackrel{0}{W_{2}^{1}}(\square)$ that consists of continuous piecewise linear functions.

Theorem 4.1. Under the assumptions made above in this section, we have

$$
\left\|u-u_{h}\right\|_{W_{2}^{1}(\square)} \leq C h^{\alpha}\left(\|u\|_{W_{2}^{1+\alpha}(\mathcal{D})}+\frac{1}{\omega}\|\phi\|_{\left(W_{2}^{\alpha}\left(\mathcal{D}^{\perp}\right)\right)^{2}}\right),
$$

where $u$ is the exact solution and $u_{h}$, earlier denoted by $v$, the FEM solution. Here and below $C$ denotes a generic constant which depends only on the domains $\square$ and $\mathcal{D}$.

Proof. The proof requires several steps, formulated as lemmas.

We first need to represent equation (4.1) as in (3.1).

Lemma 4.1. Let $\stackrel{0}{W_{2}^{1}}(\square)$ be the Hilbert space with the scalar product

$$
(u, v)=(u, v)_{W_{2}^{0}(\square)}^{0}=\int_{\square} \operatorname{grad} u \cdot \operatorname{grad} v d \square,
$$


and let the operator $A$ be defined by

$$
\int_{\mathcal{D}} \operatorname{grad} u \cdot \operatorname{grad} v d \mathcal{D}=(A u, v), \forall u, v \in \stackrel{0}{W}_{2}^{1}(\square) .
$$

Let further the right-hand side $f$ be defined by

$$
\int_{\square} \phi \cdot \operatorname{grad} v d \square=(f, v), \forall v \in \stackrel{0}{W}_{2}^{1}(\square) .
$$

Then the weak form of equation (4.1),

$$
\int_{\square}(k+\omega) \operatorname{grad} u \cdot \operatorname{grad} v d \square=\int_{\square} \phi \cdot \operatorname{grad} v d \square, \forall v \in \stackrel{0}{W_{2}^{1}}(\square)
$$

is equivalent to our original operator equation $(A+\omega I) u=f$, written in the following form

$$
((A+\omega I) u, v)=(f, v), \forall v \in \stackrel{0}{W_{2}^{1}}(\square) .
$$

Proof. From our definition of $A$,

$$
\begin{aligned}
\int_{\square}(k & +\omega) \operatorname{grad} u \cdot \operatorname{grad} v d \square \\
& =\int_{\mathcal{D}} \operatorname{grad} u \cdot \operatorname{grad} v d \mathcal{D}+\omega \int_{\square} \operatorname{grad} u \cdot \operatorname{grad} v d \square=((A+\omega I) u, v),
\end{aligned}
$$

or, in other words,

$$
A+\omega I=(\Delta)^{-1} \operatorname{div}(k+\omega) \operatorname{grad},
$$

where $\Delta \equiv$ div grad refers to the Laplacian, defined on functions in $\stackrel{0}{W}_{2}^{1}(\square)$.

Our definition of the right-hand side $f$ is equivalent to

$$
f=\Delta^{-1} \operatorname{div} \phi \text {. }
$$

The statement of the lemma follows immediately.

The next important step, simple for our example, is to characterize the subspaces $\operatorname{Ker}(A)$ and $\operatorname{Im}(A)$. We use the definition of the operator $A$ directly.

Lemma 4.2. The subspace $\operatorname{Ker}(A)$ is $\stackrel{0}{W_{2}^{1}}\left(\mathcal{D}^{\perp}\right)$ extended by zero to $\mathcal{D}$. The subspace $\operatorname{Im}(A)$ is the set of all functions in $W_{2}^{1}(\square)$ which are harmonic in $\mathcal{D}^{\perp}$.

This result allows us to describe $u_{1}$ and $f_{0}$, which play main roles in our analysis in Section 3, immediately.

Corollary 4.1. The function $u_{1}$ is the restriction of the function $u$ to the subdomain $\mathcal{D}$ extended harmonically into $\mathcal{D}^{\perp}$.

Proof. The formula $u=u_{1}+f_{0} / \omega$ of Lemma 2.2 gives an orthogonal sum, with $u_{1} \in \operatorname{Im}(A)$.

Similarly, as $f=f_{0}+f_{1}$ is an orthogonal sum with $f_{0} \in \operatorname{Ker}(A)$ and $f_{1} \in \operatorname{Im}(A)$, we obtain

Corollary 4.2. The function $f_{1}$ is the restriction of $f$ to the subdomain $\mathcal{D}$ extended harmonically into $\mathcal{D}^{\perp}$. Then, $f_{0}=f-f_{1} \in W_{2}^{1}\left(\mathcal{D}^{\perp}\right)$, extended by zero in $\mathcal{D}$. 
Remark 4.1. The structure of the subspace $\operatorname{Ker}(A)$ and, thus, that of $\operatorname{Im}(A)$, the orthogonal complement of the former, clearly depends on topological properties of our domain $\square$ and its subdomain $\mathcal{D}$. For example, if mes $\{\partial \mathcal{D} \cap \partial \square\}=0$, contrary to our assumption, $\operatorname{Ker}(A)$ would consist of functions, taking a constant, not necessarily zero, value in $\mathcal{D}$. The situation gets even more complex, if $\mathcal{D}$ is not connected; see [3]. In the present paper, we consider only the simplest possible scenario.

In Section 2 we have established that our abstract problem, though not formally well posed itself, can be split into two well posed problems, under assumption (2.2). We follow [3] to prove that assumption (2.2) holds for equation (4.1) under our assumptions on the subdomains. We do it in two steps using the following

Proposition 4.1. There exists a constant $\kappa>0$ such that for any function $v \in$ $\stackrel{0}{W_{2}^{1}}(\square)$ there is a function $w \in \stackrel{0}{W_{2}^{1}}(\square)$ satisfying $w-v \in \operatorname{Ker}(A)$ and

$$
\int_{\mathcal{D}} \operatorname{grad} w \cdot \operatorname{grad} w d \mathcal{D} \geq \kappa \int_{\square} \operatorname{grad} w \cdot \operatorname{grad} w d \square .
$$

Lemma 4.3. Proposition 4.1 holds under our assumptions on the subdomains.

Proof. The proposition is, in fact, a well known extension theorem in $W_{2}^{1}(\square)$ from the polygon $\mathcal{D}$ to the polygon $\square$. It holds for general Lipschitz domains as well; see, e.g., [10, 32].

Lemma 4.4. Assumption (2.2) is equivalent to Proposition 4.1, where $c=\kappa$.

Proof. Assumption (2.2) means that

$$
(A v, v) \geq c(v, v), v \in \mathbf{I m}(A) .
$$

By the definition of $A$,

$$
k_{0} \int_{\mathcal{D}} \operatorname{grad} v \cdot \operatorname{grad} v d \mathcal{D} \leq \int_{\mathcal{D}} \operatorname{grad} v \cdot \operatorname{grad} v d \mathcal{D}=(A v, v) .
$$

Thus, we just need to prove that the inequality of the proposition is equivalent to

$$
\int_{\mathcal{D}} \operatorname{grad} v \cdot \operatorname{grad} v d \mathcal{D} \geq \kappa \int_{\square} \operatorname{grad} v \cdot \operatorname{grad} v d \square, v \in \mathbf{I m}(A) .
$$

Let us suppose that the proposition holds. We apply inequality (4.4) to a function $v \in \operatorname{Im}(A)$, using the fact that $v=w$ in $\mathcal{D}$ since $w-v \in \operatorname{Ker}(A)$ :

$$
\int_{\mathcal{D}} \operatorname{grad} v \cdot \operatorname{grad} v d \mathcal{D} \geq \kappa \int_{\square} \operatorname{grad} w \cdot \operatorname{grad} w d \square .
$$

Finally,

$$
\begin{array}{r}
\int_{\square} \operatorname{grad} w \cdot \operatorname{grad} w d \square=(w, w)=(v, v)+ \\
(w-v, w-v) \geq(v, v) \\
=\int_{\square} \operatorname{grad} v \cdot \operatorname{grad} v d \square,
\end{array}
$$

as $w=v+(w-v)$ is an orthogonal decomposition. 
To prove the reverse, let us consider an arbitrary function $v \in \stackrel{0}{W_{2}^{1}}(\square)$ and represent it as an orthogonal decomposition $v=w+(v-w), w \in \operatorname{Im}(A), v-w \in$ $\operatorname{Ker}(A)$. Then (4.5) holds for $w \in \operatorname{Im}(A)$ and (4.4) immediately follows.

Remark 4.2. We note that the proposition is not true, in general, if the boundary of $\mathcal{D}$ is not Lipschitz, cf., e.g., 3]. For periodic boundary value problems, extension theorems can be found in 34,3 .

The previous step guarantees, by Lemmas 2.1,2.3, that equation (2.1) for $u_{1}$ is well-posed. We now have to prove that the functions $u_{1}$ and $f_{0}$, of Theorem 3.1 are smooth enough (uniformly in $\omega$ ) in $\mathcal{D}$ and $\mathcal{D}^{\perp}$ under natural assumptions, so that we can use standard FEM approximation results.

We first consider $f_{0}$ :

Lemma 4.5. Let $\phi \in\left(W_{2}^{\alpha}\left(\mathcal{D}^{\perp}\right)\right)^{2}$ for a positive constant $\alpha<1 / 2$. Then $f_{0}$ also has extra smoothness:

$$
\left\|f_{0}\right\|_{W_{2}^{1+\alpha}\left(\mathcal{D}^{\perp}\right)} \leq C\|\phi\|_{\left(W_{2}^{\alpha}\left(\mathcal{D}^{\perp}\right)\right)^{2}} .
$$

Proof. $f_{0} \in \operatorname{Ker}(A)$ can be defined by

$$
\left(f-f_{0}, v\right)=0, \forall v \in \operatorname{Ker}(A),
$$

i.e., $f_{0}$ vanishes in $\mathcal{D}$ and its restriction to $\mathcal{D}^{\perp}$ satisfies $f_{0} \in \stackrel{0}{W_{2}^{1}}\left(\mathcal{D}^{\perp}\right)$ and

$$
\int_{\mathcal{D}^{\perp}}\left(\operatorname{grad} f_{0}-\phi\right) \cdot \operatorname{grad} v d \mathcal{D}^{\perp}=0, \forall v \in \stackrel{0}{1}\left(\mathcal{D}_{2}^{\perp}\right) ;
$$

see Corollary 4.2 Under our assumptions on $\mathcal{D}^{\perp}$, this problem is well posed:

$$
\left\|f_{0}\right\|_{W_{2}^{1}\left(\mathcal{D}^{\perp}\right)} \leq C\|\phi\|_{\left(L_{2}\left(\mathcal{D}^{\perp}\right)\right)^{2}} .
$$

Moreover, if the function $\phi$ is smooth in $\mathcal{D}^{\perp}$, the statement of the lemma follows from well-known regularity results (e.g., 21, 26, 32]) as $f_{0}$ is a solution of the Poisson equation in $W_{2}^{1}\left(\mathcal{D}^{\perp}\right)$.

By Lemmas 2.2 2.3,

$$
\left\|u_{1}\right\|_{W_{2}^{1}(\square)} \leq C\|\phi\|_{\left(L_{2}(\mathcal{D})\right)^{2}}
$$

i.e., the function $u_{1}$ is bounded in $W_{2}^{1}(\square)$, uniformly in $\omega$. We also need $u_{1}$ to be smooth enough in $\mathcal{D}$ and $\mathcal{D}^{\perp}$, uniformly in $\omega$ :

Lemma 4.6. If $u \in W_{2}^{1+\alpha}(\mathcal{D})$ for a positive constant $\alpha<1 / 2$, then

$$
\left\|u_{1}\right\|_{W_{2}^{1+\alpha}(\mathcal{D})}+\left\|u_{1}\right\|_{W_{2}^{1+\alpha}\left(\mathcal{D}^{\perp}\right)} \leq C\|u\|_{W_{2}^{1+\alpha}(\mathcal{D})} .
$$

Proof. By Corollary 4.1, the function $u_{1}$ is simply the restriction of the function $u$ to the subdomain $\mathcal{D}$ extended harmonically into $\mathcal{D}^{\perp}$. Thus, as $u \in W_{2}^{1+\alpha}(\mathcal{D})$, we have trivially

$$
\left\|u_{1}\right\|_{W_{2}^{1+\alpha}(\mathcal{D})}=\|u\|_{W_{2}^{1+\alpha}(\mathcal{D})} \cdot
$$

Moreover,

$$
\left\|u_{1}\right\|_{W_{2}^{1+\alpha}\left(\mathcal{D}^{\perp}\right)} \leq C\|u\|_{W_{2}^{1+\alpha}(\mathcal{D})} .
$$

The latter statement is based on the fact that $u_{1}$ is harmonic in $\mathcal{D}^{\perp}$ and is an extension of the function $u$, which is smooth in $\mathcal{D}$. Thus, $u_{1}$ solves the Laplace 
equation with nonhomogeneous Dirichlet boundary condition on $\partial \mathcal{D}^{\perp}$ given by the trace of $u$ on $\partial \mathcal{D}^{\perp}$. Under our assumption that $\mathcal{D}^{\perp}$ is a polygon strictly inside of $\square$, we have

$$
\left.u\right|_{\partial \mathcal{D}^{\perp}} \in W_{2}^{1 / 2+\alpha}\left(\partial \mathcal{D}^{\perp}\right)
$$

(e.g., [26, 32]). Then, the required estimate follows from known regularity results for general Lipschitz domains (e.g., [26, 40, 32]).

We now turn our attention to the finite element method. Let $P$ be the $\stackrel{0}{W}_{2}^{1}(\square)$ orthogonal projector onto $\operatorname{Im}(P)$, which is our FEM subspace of $\stackrel{0}{W_{2}^{1}}(\square)$ that consists of continuous piecewise linear functions on a standard finite element triangulation, with shape regular and quasi-uniform triangles. Then our abstract Ritz-Galerkin method becomes a standard FEM.

The subspace $\operatorname{Ker}(B)$ is, by Lemma 3.2 a subspace of continuous piecewise linear functions in $\operatorname{Im}(P)$ which vanish on $\mathcal{D}$. The subspace $\operatorname{Im}(B)$ consists of functions in $\operatorname{Im}(P)$ that are discrete harmonic on $\mathcal{D}^{\perp}$.

Based on our regularity result, Lemma 4.5, $f_{0}$ can be approximated in $\stackrel{0}{W_{2}^{1}}\left(\mathcal{D}^{\perp}\right)$ by a finite element-based subspace:

\section{Lemma 4.7.}

$$
\operatorname{dist}\left\{f_{0} ; \mathbf{I m}(P) \cap \operatorname{Ker}(A)\right\} \leq C h^{\alpha}\|\phi\|_{\left(W_{2}^{\alpha}\left(\mathcal{D}^{\perp}\right)\right)^{2}} .
$$

Proof. We recall that the subspace $\operatorname{Ker}(A)$ is $\stackrel{0}{W_{2}^{1}}\left(\mathcal{D}^{\perp}\right)$ extended by zero to $\mathcal{D}$. By our assumption that none of finite elements cuts the interface between $\mathcal{D}$ and $\mathcal{D}^{\perp}$, the intersection $\operatorname{Im}(P) \cap \operatorname{Ker}(A)$ is simply a subspace of our FEM space of FEM functions taking zero value in $\mathcal{D}$. As the function $f_{0}$ is in $W_{2}^{1}\left(\mathcal{D}^{\perp}\right)$, the distance we need to estimate is the standard $\stackrel{0}{W_{2}^{1}}\left(\mathcal{D}^{\perp}\right)$ FEM approximation error in $\mathcal{D}^{\perp}$ and the estimate follows from a standard result of FEM theory; see, e.g., [13].

Similarly, by Lemma 4.6. $u_{1}$ can be approximated by a finite element-based subspace:

\section{Lemma 4.8.}

$$
\operatorname{dist}\left\{u_{1} ; \operatorname{Im}(P)\right\} \leq C h^{\alpha}\|u\|_{W_{2}^{1+\alpha}(\mathcal{D})} .
$$

Now we need to prove that assumption (3.4) holds in our example. As in the continuous case discussed earlier in the proof, we do it in two steps, using the following

Proposition 4.2. There exists a constant $\tilde{\kappa}>0$ such that for any function $v \in$ $\stackrel{0}{W_{2}^{1}}(\square) \cap \operatorname{Im}(P)$ there exists a function $w \underset{0}{ } w \stackrel{W_{2}^{1}}{(\square)} \cap \operatorname{Im}(P)$ satisfying $w-v \in$ $\operatorname{Ker}(A) \cap \operatorname{Im}(P)$ and

$$
\int_{\mathcal{D}} \operatorname{grad} w \cdot \operatorname{grad} w d \mathcal{D} \geq \tilde{\kappa} \int_{\square} \operatorname{grad} w \cdot \operatorname{grad} w d \square .
$$

Remark 4.3. Let us note that Proposition 4.2 always holds for any FEM, since a FEM uses a finite dimensional trial subspace. However, in some cases the constant, $\tilde{\kappa}$, goes to zero with $h$, the mesh size parameter, even if the continuous analog, 
Proposition 4.1, holds. Then $\tilde{c}$ decreases too, which affects our error estimate for small $\omega$. Therefore, if possible, the FEM should be constructed such that $\tilde{\kappa}$ in Proposition 4.2 can be chosen to be independent of $h$.

The following well-known lemma (see, e.g., [1, 42]) provides the required result for our example.

Lemma 4.9. Under the assumption made earlier that none of finite elements cut the boundary $\partial \mathcal{D} \cup \partial \mathcal{D}^{\perp} \cup \partial \square$, Proposition 4.2 holds with a constant $\tilde{\kappa}$ independent of $h$.

We now prove that assumption (3.4) is equivalent to this finite element extension theorem.

Lemma 4.10. Assumption (3.4) is equivalent to Proposition [4.2, in particular, Proposition 4.2 justifies assumption (3.4) with $\tilde{c}=\tilde{\kappa}$.

Proof. Assumption (3.4) means that

$$
(B v, v) \geq \tilde{c}(v, v), v \in \mathbf{I m}(B) .
$$

By the definition of $B$, we have $(A v, v)=(B v, v)$. Then, by (4.2),

$$
\int_{\mathcal{D}} \operatorname{grad} v \cdot \operatorname{grad} v d \mathcal{D}=(B v, v) .
$$

Thus, we just need to prove that the inequality of the proposition is equivalent to

$$
\int_{\mathcal{D}} \operatorname{grad} v \cdot \operatorname{grad} v d \mathcal{D} \geq \tilde{\kappa} \int_{\square} \operatorname{grad} v \cdot \operatorname{grad} v d \square, v \in \mathbf{I m}(B) .
$$

Let us suppose that the proposition holds. We can then apply inequality (4.6) to a function $v \in \operatorname{Im}(B)$, using the fact that $v=w$ in $\mathcal{D}$ since $w-v \in \operatorname{Ker}(B) \subset$ $\operatorname{Ker}(A)$ :

$$
\int_{\mathcal{D}} \operatorname{grad} v \cdot \operatorname{grad} v d \mathcal{D} \geq \tilde{\kappa} \int_{\square} \operatorname{grad} w \cdot \operatorname{grad} w d \square .
$$

Finally,

$$
\begin{array}{r}
\int_{\square} \operatorname{grad} w \cdot \operatorname{grad} w d \square=(w, w)=(v, v)+ \\
(w-v, w-v) \geq(v, v) \\
=\int_{\square} \operatorname{grad} v \cdot \operatorname{grad} v d \square,
\end{array}
$$

as $w=v+(w-v)$ is an orthogonal decomposition.

To prove the reverse, let us consider an arbitrary function $v \in \operatorname{Im}(P)$ and represent it as an orthogonal decomposition $v=w+(v-w), w \in \operatorname{Im}(B), v-w \in$ $\operatorname{Ker}(B)$. Then we have (4.7) for $w \in \mathbf{I m}(B)$ and (4.4) immediately follows.

Thus, our constant $\tilde{c}$ does not depend on $\omega$ and $h$. Another needed quantity, the norm $\|(I-P) A P\|$, can be estimated trivially:

$$
\|(I-P) A P\| \leq\|A\| \leq 1,
$$

and therefore, $\|(I-P) A P\|$ is bounded uniformly in $\omega$ as well.

Putting all the arguments above together turns our abstract estimate (3.10) into the error estimate (4.3), we needed to prove. 
We finally consider the question of regularity of the solution $u$, uniform in $\omega$. Theorem 4.1 involves a term $\|u\|_{W_{2}^{1+\alpha}(\mathcal{D})}$. We will argue that this quantity is uniformly bounded in $\omega$, with a fixed positive $\alpha$ up to a certain value $\alpha_{\max }$, in the following sense:

$$
\|u\|_{W_{2}^{1+\alpha}(\mathcal{D})} \leq C\|\phi\|_{\left(W_{2}^{\alpha}(\square)\right)^{2}}, \alpha \in\left(0, \alpha_{\max }\right) .
$$

The crucial value $\alpha_{\max }$ is determined by the strongest possible singularity in the solution $u$, informally speaking. It is well-known that, under our assumptions, the solution $u$ is regular strictly inside of subdomains $\mathcal{D}$ and $\mathcal{D}^{\perp}$ for a sufficiently smooth right-hand side $\phi$, but may have singularities near the boundary $\partial \square$ and near the interface

$$
\Gamma=\partial \mathcal{D} \cap \partial \mathcal{D}^{\perp}
$$

defined by the jump in the coefficients. The interface $\Gamma$ simply separates subdomains $\mathcal{D}$ and $\mathcal{D}^{\perp}$, where the diffusion coefficient takes different values.

Regularity of the solution of the problem of the present section is closely related to regularity of transmission and diffraction problems and properties of layer potentials and Steklov-Poincaré operators on nonsmooth interfaces, which have been extensively studied; see, e.g., recent papers [40, 33], the book [32], and references therein. A very brief summary of relevant known results follows.

Two major cases need to be considered separately: when domain $\mathcal{D}^{\perp}$ is strictly inside (SI) of $\square$ and the opposite case, when it is not (NSI). The former, the SI, case is simpler as it can be reduced to studying layer potentials on $\Gamma$ without boundary conditions, because in the SI case the boundary $\Gamma$ is a closed Lipschitz curve without selfintersection and it does not have any common points, also called junction points, with the boundary $\partial \square$, where the homogeneous Dirichlet boundary condition is enforced.

Under the assumption that both subdomains are polygons, the only possible singularities are known to be vertex singularities corresponding to corner points of $\partial \square$ and $\Gamma$, and, in the NSI case, to junction points of $\partial \square$ and $\Gamma$. These singularities can be calculated explicitly, using well-known equations for $\alpha_{\max }$ for every vertex; see, e.g., Kellogg in [25], p. 112, who shows that $\alpha_{\max } \in[1 / 2,3 / 2]$ for the SI case. The NSI case is also affected by the type of boundary conditions imposed on the boundary $\partial \square$. For our case of the Dirichlet boundary conditions everywhere on $\partial \square$, [33] states on p. 368 that $\alpha_{\max } \in[1 / 4,1]$. Let us highlight that, though the actual singularity due to the jump in coefficients does depend on $\omega$, the lower bounds, $1 / 2$ for the SI case, and $1 / 4$ for the NSI case, do not, thus providing regularity uniform in $\omega$. An important issue of uniformity of constants in regularity estimates is not considered in [25, 33, therefore leaving the question of validity of estimate (4.8) open.

For general Lipschitz domains instead of polygons, the situation is even more delicate. None of publications known to us specifically addresses regularity issues uniformly in the jump of coefficients. The SI case is treated (e.g., in [19]) from where one can derive (4.8) with $\alpha_{\max }=1 / 2$, but for a fixed $\omega$. A similar result for the NSI case is obtained in [40, Theorem 5, with an extra assumption on the junction points of $\partial \square$ with $\Gamma$. The latter result does not appear to be uniform in $\omega$ as it would contradict a sharp lower bound $1 / 4$ for polygons cited above. In the former result of [19], however, for the SI case, the dependence on $\omega$ can be removed, as we show in the next theorem, using a different technique. We note that in the 
limit $\omega=0$, which corresponds to a Neumann boundary value problem on $\mathcal{D}$, the regularity result holds with $\alpha_{\max }=1 / 2$; see, e.g., [26]. We will provide a sketch of the proof of uniform regularity for the SI case and general Lipschitz domains in the rest of the section. Let us just remind the reader that we have already made the SI assumption in the beginning of the section and used it in Lemma 4.6 .

Theorem 4.2. Let the assumptions of Theorem 4.1 be satisfied, except that the domain $\square$ and its subdomains $\mathcal{D}$ and $\mathcal{D}^{\perp}$ can be general Lipschitz domains, not necessarily polygons. Then estimate (4.8) holds for $\alpha_{\max }=1 / 2$, uniformly in $\omega$.

Proof. We first simplify equation (4.1) by introducing a new function

$$
w=u-\hat{u} \in \stackrel{0}{W_{2}^{1}}(\square),
$$

where function $\hat{u} \in \stackrel{0}{W}_{2}^{1}(\square)$ satisfies the equation

$$
(1+\omega) \int_{\mathcal{D}}(\operatorname{grad} \hat{u}-\phi) \cdot \operatorname{grad} v d \mathcal{D}=0, \forall v \in \stackrel{0}{W_{2}^{1}}(\mathcal{D}),
$$

in $\mathcal{D}$ and satisfies the equation

$$
\omega \int_{\mathcal{D}^{\perp}}(\operatorname{grad} \hat{u}-\phi) \cdot \operatorname{grad} v d \mathcal{D}^{\perp}=0, \forall v \in \stackrel{0}{W}_{2}^{1}\left(\mathcal{D}^{\perp}\right),
$$

in $\mathcal{D}^{\perp}$.

Let us introduce the following notation for the corresponding restrictions $\hat{u}_{\mathcal{D}}=$ $\left.\hat{u}\right|_{\mathcal{D}}$ and $\hat{u}_{\mathcal{D} \perp}=\left.\hat{u}\right|_{\mathcal{D} \perp}$ of the function $\hat{u}$ and similarly for the function $\phi$. We note that equations (4.9) and (4.10) are uniformly elliptic homogeneous Dirichlet boundary value problems on Lipschitz domains. Therefore,

$$
\left\|\hat{u}_{\mathcal{D}}\right\|_{W_{2}^{1+\alpha}(\mathcal{D})} \leq C\left\|\phi_{\mathcal{D}}\right\|_{\left(W_{2}^{\alpha}(\mathcal{D})\right)^{2}}, \alpha \in\left(0, \alpha_{\max }\right)
$$

and

$$
\left\|\hat{u}_{\mathcal{D}^{\perp}}\right\|_{W_{2}^{1+\alpha}\left(\mathcal{D}^{\perp}\right)} \leq C \frac{1}{\omega}\left\|\phi_{\mathcal{D}^{\perp}}\right\|_{\left(W_{2}^{\alpha}\left(\mathcal{D}^{\perp}\right)\right)^{2}}, \alpha \in\left(0, \alpha_{\max }\right),
$$

uniformly in $\omega$ by well-known regularity results; see, e.g., 21, 22. We just now need to prove uniform regularity of $w$.

By construction, the function $w$ is piece-wise harmonic, i.e., the function $w_{\mathcal{D}}=$ $\left.w\right|_{\mathcal{D}}$ is harmonic in $\mathcal{D}$ and the function $w_{\mathcal{D}^{\perp}}=\left.w\right|_{\mathcal{D}^{\perp}}$ is harmonic in $\mathcal{D}^{\perp}$. On the interface of the jump in the coefficients, $\Gamma$, the functions $w_{\mathcal{D}}$ and $w_{\mathcal{D}^{\perp}}$ have the same trace $\left.w\right|_{\Gamma}$ from $\mathcal{D}$ and $\mathcal{D}^{\perp}$ and satisfy the following standard transmission condition on $\Gamma$ between $\mathcal{D}$ and $\mathcal{D}^{\perp}$ for the normal components:

$$
\left.\mathbf{n} \cdot\left((1+\omega) \operatorname{grad}\left(w_{\mathcal{D}}+\hat{u}_{\mathcal{D}}\right)-\phi_{\mathcal{D}}\right)\right|_{\Gamma}=\left.\mathbf{n} \cdot\left(\omega \operatorname{grad}\left(w_{\mathcal{D}^{\perp}}+\hat{u}_{\mathcal{D}^{\perp}}\right)-\phi_{\mathcal{D}^{\perp}}\right)\right|_{\Gamma}
$$

where the left side corresponds to $\mathcal{D}$ and the right side to $\mathcal{D}^{\perp}$, and $\mathbf{n}$ is the normal direction on $\Gamma$, oriented outward $\mathcal{D}$.

Let us rewrite equation (4.13) by collecting all terms with $w$ in the left-hand side:

$$
\begin{aligned}
(1+ & \omega)\left.\frac{\partial}{\partial \mathbf{n}} w_{\mathcal{D}}\right|_{\Gamma}-\left.\omega \frac{\partial}{\partial \mathbf{n}} w_{\mathcal{D}^{\perp}}\right|_{\Gamma} \\
& =\left.\mathbf{n} \cdot\left(\phi_{\mathcal{D}}-(1+\omega) \operatorname{grad} \hat{u}_{\mathcal{D}}\right)\right|_{\Gamma}-\left.\mathbf{n} \cdot\left(\phi_{\mathcal{D}^{\perp}}-\omega \operatorname{grad} \hat{u}_{\mathcal{D}^{\perp}}\right)\right|_{\Gamma} .
\end{aligned}
$$


Let us now define a Steklov-Poincaré operator $S_{\mathcal{D}}$ that, for a given harmonic function $w_{\mathcal{D}}=\left.w\right|_{\mathcal{D}}$, where $w \in \stackrel{0}{W}_{2}^{1}(\square)$, maps its trace on $\Gamma$ into its Neumann datum on $\Gamma$ :

$$
S_{\mathcal{D}}:\left.\left.w_{\mathcal{D}}\right|_{\Gamma} \mapsto \frac{\partial}{\partial \mathbf{n}} w_{\mathcal{D}}\right|_{\Gamma}
$$

Let us define, similarly, the Steklov-Poincaré operator $S_{\mathcal{D}^{\perp}}$ for the other domain $\mathcal{D}^{\perp}$ using the normal direction on $\Gamma$, oriented outwards from $\mathcal{D}^{\perp}$. Steklov-Poincaré operators act between the corresponding space of trace functions

$$
\Lambda=\left\{\left.w\right|_{\Gamma}, \forall w \underset{\sim}{W_{2}^{1}}(\square)\right\} .
$$

and its dual $\Lambda^{\prime}$. These operators are symmetric and positive semi-definite on $\Lambda$ and bounded as mappings $\Lambda \rightarrow \Lambda^{\prime}$; see, e.g., 37]. Moreover, the operator $S_{\mathcal{D}}$ is positive definite, because of the homogeneous Dirichlet boundary conditions on $\partial \square$.

As the domain $\mathcal{D}^{\perp}$ is strictly inside, i.e., $\Gamma$ is a closed curve, the space $\Lambda$ is simply $W_{2}^{1 / 2}(\Gamma)$ and $\Lambda^{\prime}$ is $W_{2}^{-1 / 2}(\Gamma)$.

Using the Steklov-Poincaré operators just defined, we rewrite equation (4.14) as

$$
\left((1+\omega) S_{\mathcal{D}}+\omega S_{\mathcal{D}^{\perp}}\right) \lambda=\chi
$$

where we introduce new notations: $\lambda$ for the common trace $\left.w\right|_{\Gamma}$ of $w_{\mathcal{D}}$ and $w_{\mathcal{D}^{\perp}}$ on $\Gamma$ and $\chi$ for the right-hand side of (4.14). The operator $(1+\omega) S_{\mathcal{D}}+\omega S_{\mathcal{D}^{\perp}} \geq S_{\mathcal{D}}$ is symmetric, positive definite and bounded, uniformly in $\omega, 0 \leq \omega \leq 1$, e.g., [5]. The right-hand side $\chi$ is bounded uniformly in $\omega$, i.e.,

$$
\|\chi\|_{W_{2}^{-1 / 2}(\Gamma)} \leq C\|\phi\|_{\left(L_{2}(\square)\right)^{2}}
$$

as follows from its definition; see, e.g., [32]. Therefore, the solution $\lambda$ is bounded uniformly in $W_{2}^{1 / 2}(\Gamma)$, i.e.,

$$
\|\lambda\|_{W_{2}^{1 / 2}(\Gamma)} \leq C\|\phi\|_{\left(L_{2}(\square)\right)^{2}}
$$

which leads to uniform boundedness of $w$ and $u_{1}$ in $\stackrel{0}{W_{2}^{1}}(\square)$. This is the result established earlier in the paper using Lemmas 2.2 2.3 Thus, the restriction of $u$ to $\mathcal{D}$ is uniformly bounded in $W_{2}^{1}(\mathcal{D})$.

At this point, we are prepared to study the uniform regularity of $\lambda$, which shall demonstrate uniform regularity of $u$ in $\mathcal{D}$.

We first notice that $\chi \in W_{2}^{-1 / 2+\alpha}(\Gamma)$ because of extra smoothness of our functions $\phi$ and $\hat{u}$ that determine $\chi$ according to (4.11), (4.12), and (4.14):

$$
\|\chi\|_{W_{2}^{-1 / 2+\alpha}(\Gamma)} \leq C\|\phi\|_{\left(W_{2}^{\alpha}(\square)\right)^{2}} .
$$

In recent works [14, 23, 32], a regularity theory of Steklov-Poincaré operators is established for Lipschitz domains. Using these results for our situation, we have that for all $\alpha \in[0,1 / 2)$ the Steklov-Poincaré operator $S_{\mathcal{D}^{\perp}}$ is bounded as

$$
S_{\mathcal{D}^{\perp}}: W_{2}^{1 / 2+\alpha}(\Gamma) \mapsto W_{2}^{-1 / 2+\alpha}(\Gamma)
$$

and the Steklov-Poincaré operator $S_{\mathcal{D}}$ is coercive:

$$
\left\|S_{\mathcal{D}}^{-1} \psi\right\|_{W_{2}^{1 / 2+\alpha}(\Gamma)} \leq C\left(\|\psi\|_{W_{2}^{-1 / 2+\alpha}(\Gamma)}+\left\|S_{\mathcal{D}}^{-1} \psi\right\|_{W_{2}^{1 / 2}(\Gamma)}\right) .
$$

The boundedness of $S_{\mathcal{D}}$ and the coerciveness (modulo constants) of $S_{\mathcal{D}^{\perp}}$ are not important for our further arguments. 
It is not clear, whether our Steklov-Poincaré operators are symmetric and positive semi-definite on $W_{2}^{1 / 2+\alpha}(\Gamma)$ as they are on $W_{2}^{1 / 2}(\Gamma)$, so we need to use a different approach.

Let us rewrite equation (4.15) as

$$
\left(I+\frac{\omega}{1+\omega} S_{\mathcal{D}}^{-1} S_{\mathcal{D}^{\perp}}\right) \lambda=\frac{1}{1+\omega} S_{\mathcal{D}}^{-1} \chi
$$

As the operator $S_{\mathcal{D}}^{-1} S_{\mathcal{D}^{\perp}}$ is bounded in $W_{2}^{1 / 2+\alpha}(\Gamma)$, the operator of equation (4.17) is a small perturbation of the identity, therefore, it has a bounded inverse in $W_{2}^{1 / 2+\alpha}(\Gamma)$, for nonnegative $\omega$ small enough, e.g., for

$$
0 \leq \omega \leq\left\|S_{\mathcal{D}}^{-1} S_{\mathcal{D}^{\perp}}\right\|_{W_{2}^{1 / 2+\alpha}(\Gamma)}^{-1} .
$$

Thus, the regularity estimate holds

$$
\|\lambda\|_{W_{2}^{1 / 2+\alpha}(\Gamma)} \leq C\|\phi\|_{\left(W_{2}^{\alpha}(\square)\right)^{2}}
$$

uniformly in $\omega$ satisfying (4.18).

The function $\lambda$, the trace of $w$, is uniformly bounded in $W_{2}^{1 / 2+\alpha}(\Gamma)$. Thus, the function $w_{\mathcal{D}}$, as a harmonic extension of $\lambda$ to $\mathcal{D}$, is uniformly bounded in $W_{2}^{1+\alpha}(\mathcal{D})$ :

$$
\left\|w_{\mathcal{D}}\right\|_{W_{2}^{1 / 2+\alpha}(\mathcal{D})} \leq C\|\phi\|_{\left(W_{2}^{\alpha}(\square)\right)^{2}}
$$

Indeed, $w_{\mathcal{D}}$ solves the Laplace equation in the Lipschitz domain $\mathcal{D}$ with homogeneous Dirichlet boundary conditions on $\partial \square$ and nonhomogeneous Dirichlet boundary conditions, given by $\lambda \in W_{2}^{1 / 2+\alpha}(\Gamma)$, on $\Gamma$. Thus, known regularity results (e.g., 26, 40, 32]) can be applied.

As $u=w+\hat{u}$, we combine the previous inequality with (4.11) to get (4.8) uniformly in $\omega$, satisfying (4.18).

Finally, we repeat that the statement of the theorem for a fixed positive $\omega$ follows from [19, 40]. Therefore, we can take $\omega \in[0,1]$.

\section{NUMERICAL EXPERIMENTS FOR THE DIFFUSION EQUATION}

We illustrate our theoretical estimates by solving three model problems numerically: a square divided into two rectangles (Case I), an L-shaped domain divided into two rectangles (Case II), and a square divided into four squares (Case III). See Figure 5.1
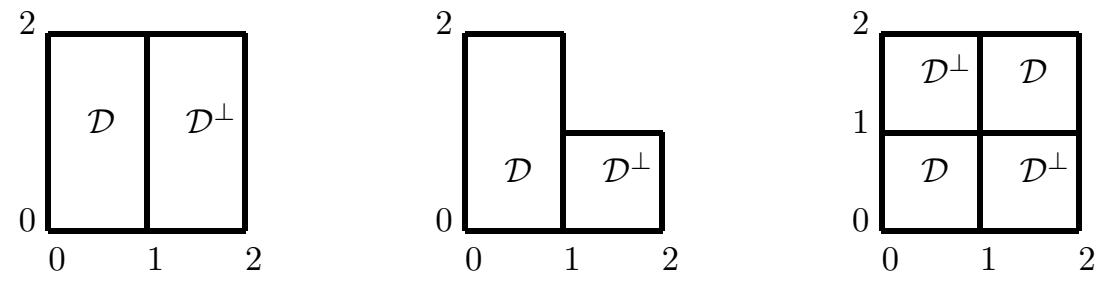

Figure 5.1. Model Domains: Cases I-III 

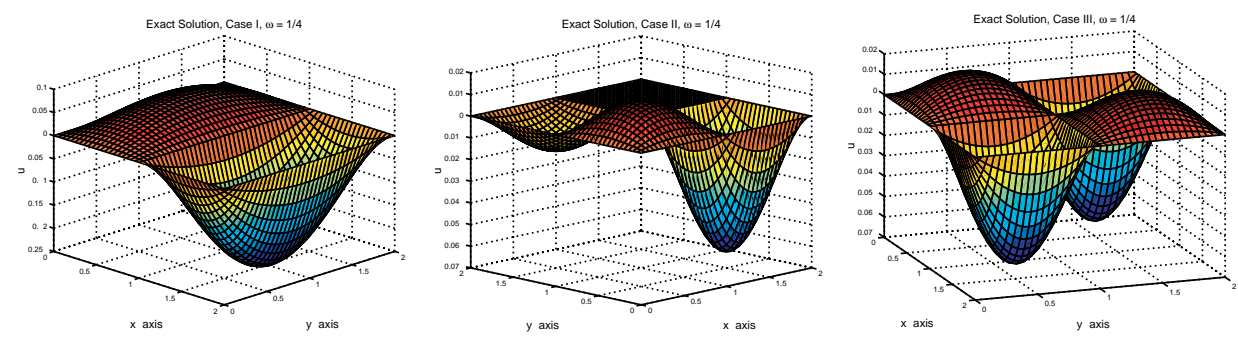

Figure 5.2. Exact Solution, $\omega=1 / 4$, Cases I-III

We note that none of these cases satisfies our assumptions on the subdomains. In Case III, even the extension theorem fails. Nevertheless, we shall see that numerical results for the solutions chosen are very favorable and support our conclusion on the uniformity of error bounds.

We assume that

$$
k=0 \text { on } \mathcal{D}^{\perp}, k=1-\omega \text { in } \mathcal{D},
$$

and that $0<\omega \leq 1$.

The exact solution is chosen to be the following function (see also Figure 5.2):

$$
\begin{gathered}
\text { Case I: } u= \begin{cases}\omega x(x-1) y(y-2) & \text { in } \mathcal{D} \\
-(x-1)(x-2) y(y-2) & \text { in } \mathcal{D}^{\perp} .\end{cases} \\
\text { Case II: } u= \begin{cases}\omega x(x-1) y(y-1) & \text { in } \mathcal{D}, 0 \leq y \leq 1 \\
-\omega x(x-1)(y-1)(y-2) & \text { in } \mathcal{D}, 1 \leq y \leq 2 \\
-(x-1)(x-2) y(y-1) & \text { in } \mathcal{D}^{\perp} .\end{cases} \\
\text { Case III: } u= \begin{cases}\omega x(x-1) y(y-1) & \text { in } \mathcal{D}, 0 \leq x \leq 1,0 \leq y \leq 1 \\
\omega(x-1)(x-2)(y-1)(y-2) & \text { in } \mathcal{D}, 1 \leq x \leq 2,1 \leq y \leq 2 \\
-(x-1)(x-2) y(y-1) & \text { in } \mathcal{D}^{\perp}, 1 \leq x \leq 2,0 \leq y \leq 1 \\
-x(x-1)(y-1)(y-2)) & \text { in } \mathcal{D}^{\perp}, 0 \leq x \leq 1,1 \leq y \leq 2\end{cases}
\end{gathered}
$$

The Galerkin method used is defined by piecewise linear functions on the standard uniform triangulation, and we use a four-point quadrature scheme (see [15]) which is exact for third-order polynomials.

The error in the approximation is computed as

$$
\epsilon=\sqrt{\int_{\square}\left|\nabla u-\nabla u_{h}\right|^{2} d \square},
$$

where $u$ is the exact solution and $u_{h}$ the approximation.

The results are shown in the figures for $1 \leq h \leq 2^{-5}$ and $1 \leq \omega \leq 2^{-5}$. Numerical results with smaller $\omega$ are practically the same as those with $\omega=2^{-5}$, within the picture resolution.

Figures 5.3 5.5 show that $\epsilon$ decreases linearly with $h$ if $\omega$ is fixed, as we expect. When $h$ is fixed, decreasing $\omega$ appears to cause $\epsilon$ to approach a limiting value. The exact solution, as well as the approximate solution, approach limits when $\omega \rightarrow 0$. Our numerical tests with $\omega=\frac{1}{32}$ and $\omega$ as small as $10^{-6}$ demonstrate that the error, $\epsilon$, approaches a limit as $\omega$ decreases. 


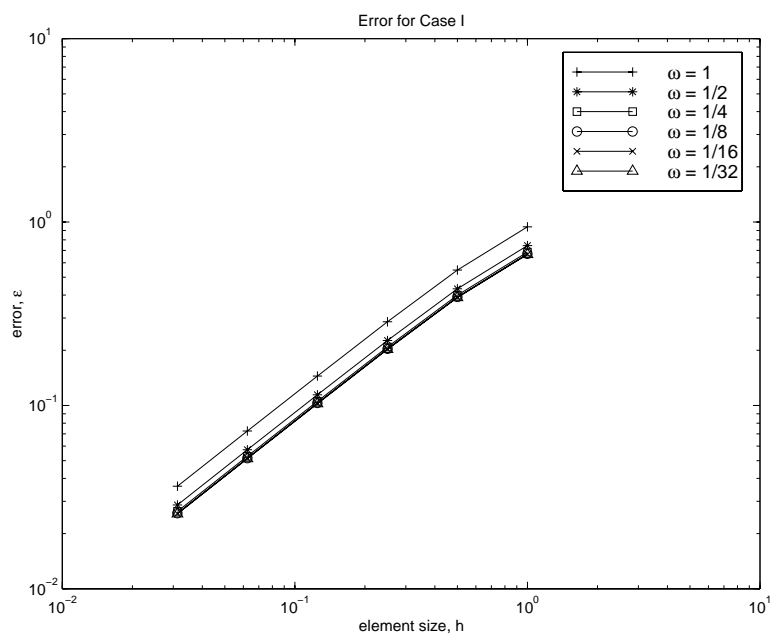

Figure 5.3. Error in Case I

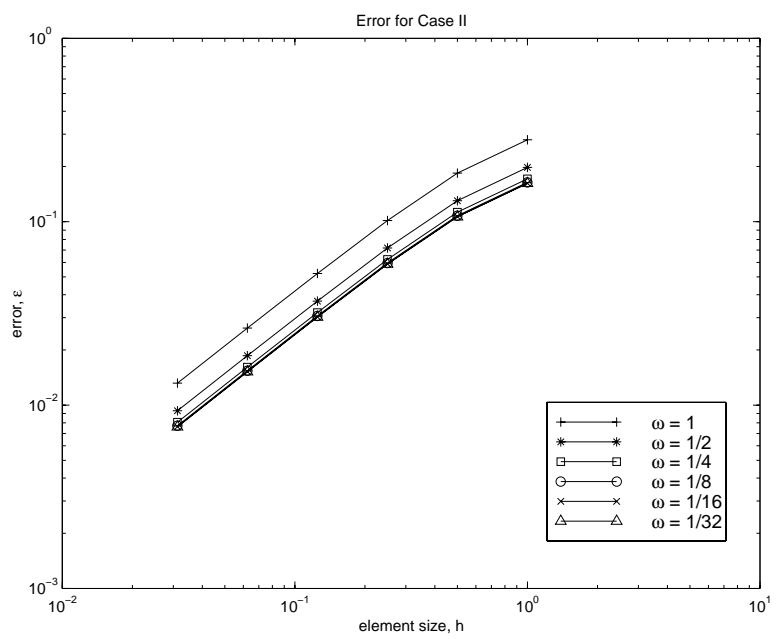

Figure 5.4. Error in Case II

In our other set of numerical tests, the solution $u$ is chosen to be independent of $\omega$. We noticed that for some functions $u$, the FEM solutions $u_{h}$ are also independent of $\omega$, and, trivially, the approximation error does not depend on $\omega$ either. We finally found a simple function

$$
u=(x+y) x(2-x) y(2-y)
$$

which in Case I led to the FEM solution $u_{h}$ changing with $\omega$. The approximation error for this case is given in Table 5.1 


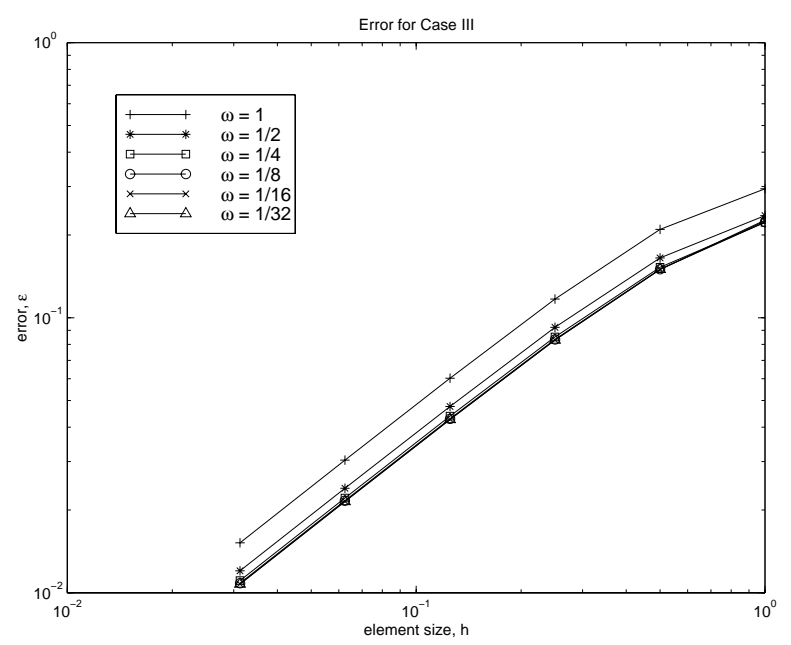

Figure 5.5. Error in Case III

TABLE 5.1. Error for Case I, exact solution $u=(x+y) x(2-x) y(2-y)$.

\begin{tabular}{|l|c|c|c|c|c|c|}
\hline & $\omega=1$ & $\omega=10^{-1}$ & $\omega=10^{-2}$ & $\omega=10^{-3}$ & $\omega=10^{-4}$ & $\omega=10^{-5}$ \\
\hline$h=1$ & 3.9470 & 3.9858 & 4.0025 & 4.0046 & 4.0048 & 4.0048 \\
\hline$h=1 / 2$ & 2.3012 & 2.3086 & 2.3118 & 2.3122 & 2.3122 & 2.3122 \\
\hline$h=1 / 4$ & 1.2034 & 1.2044 & 1.2049 & 1.2050 & 1.2050 & 1.2050 \\
\hline$h=1 / 8$ & 0.60877 & 0.60891 & 0.60897 & 0.60898 & 0.60898 & 0.60898 \\
\hline$h=1 / 16$ & 0.30529 & 0.30531 & 0.30531 & 0.30532 & 0.30532 & 0.30532 \\
\hline$h=1 / 32$ & 0.15276 & 0.15276 & 0.15276 & 0.15276 & 0.15276 & 0.15276 \\
\hline
\end{tabular}

\section{ACKNOWLEDGMENTS}

The authors thank Lars Wahlbin, John Osborn and an anonymous referee, who asked that the issue of uniform regularity be clarified. This has led to Theorem 4.2 in this revised version of the paper.

The authors are indebted to Bruce Kellogg, who kindly pointed out his important paper [25] on regularity of transmission problems on curves with corners and selfintersections.

Finally, the authors are grateful to CU-Denver graduate students Joe Cullen, David Duran, Bret Gunneson, Tom Huntley, and Constantine Kelesoglou, for carrying out the numerical experiments as their midterm assignment.

\section{REFERENCES}

1. G. P. Astrakhantsev, Method of fictitious domains for a second-order elliptic equation with natural boundary conditions, U.S.S.R. Computational Math. and Math. Phys. 18 (1978), $114-121$.

2. N. S. Bakhvalov and A. V. Knyazev, Efficient computation of averaged characteristics of composites of a periodic structure of essentially different materials, Soviet Math. Doklady 42 (1991), no. 1, 57-62. MR 92m:73007

3. , Fictitious domain methods and computation of homogenized properties of composites with a periodic structure of essentially different components, Numerical Methods and Applications (Gury I. Marchuk, ed.), CRC Press, Boca Raton, 1994, pp. 221-276. MR 95e:65113 
4. _ Preconditioned iterative methods in a subspace for linear algebraic equations with large jumps in the coefficients, Domain Decomposition Methods in Science and Engineering (D. Keyes and J. Xu, eds.), Contemporary Mathematics, vol. 180, American Mathematical Society, Providence, 1994, Proceedings of the Seventh International Conference on Domain Decomposition, October 27-30, 1993, held at the Pennsylvania State University, pp. 157-162. MR 95j:65042

5. N. S. Bakhvalov, A. V. Knyazev, and G. M. Kobel'kov, Iterative methods for solving equations with highly varying coefficients, Fourth International Symposium on Domain Decomposition Methods for Partial Differential Equations (Philadelphia, PA) (Roland Glowinski, Yuri A. Kuznetsov, Gérard A. Meurant, Jacques Périaux, and Olof Widlund, eds.), SIAM, 1991, pp. 197-205. CMP 91:12

6. N. S. Bakhvalov, A. V. Knyazev, and R. R. Parashkevov, Extension theorems for Stokes and Lamé equations for nearly incompressible media and their applications to numerical solution of problems with highly discontinuous coefficients, Numerical Linear Algebra with Applications. Accepted, May 2001.

7. N. S. Bakhvalov and G. P. Panasenko, Homogenization: Averaging of processes in periodic media. Mathematical problems in the mechanics of composite materials, Mathematics and Its Applications. Soviet Series, vol. 36, Kluwer Academic Publishers, Dordrecht/Boston/London, 1989. MR 92d:73002

8. A. Bakushinsky and A. Goncharsky, Ill-posed problems: Theory and applications, Mathematics and Its Applications, vol. 301, Kluwer Academic Publishers, Dordrecht/Boston/London, 1994. MR 96d:65101

9. Alain Bensoussan, Jacques-Louis Lions, and George Papanicolaou, Asymptotic analysis for periodic structures, Studies in Mathematics and its Applications, vol. 5, North-Holland Publishing Co., Amsterdam, 1978. MR 82h:35001

10. O. V. Besov, V. P. Il'in, and S. M. Nikol'skii, Integral representations of functions and imbedding theorems, vols. 1, 2, Wiley, New York, 1979. MR 80f:46030a MR 80f:46030b

11. Ch. Börgers and O. Widlund, On finite element domain imbedding methods, SIAM J. Numer. Anal. 27 (1990), no. 4, 963-978. MR 91g:65235

12. B. L. Buzbee, F. W. Dorr, J. A. George, and G. Golub, The direct solution of the discrete Poisson equation on irregular regions, SIAM J. Numer. Anal. 8 (1971), 722-736. MR 45:1403

13. Philippe G. Ciarlet, The finite element method for elliptic problems, North-Holland Publishing Co., Amsterdam, 1978, Studies in Mathematics and its Applications, Vol. 4. MR 58:25001

14. Martin Costabel, Boundary integral operators on Lipschitz domains: elementary results, SIAM J. Math. Anal. 19 (1988), no. 3, 613-626. MR 89h:35090

15. G. R. Cowper, Gaussian quadrature formulas for triangles, Int. J. Num. Meth. Eng. 7 (1973), 405-408.

16. G. C. A. DeRose and A. R. Diaz, Single scale wavelet approximations in layout optimization, Struct. Optimization 18 (1999), no. 1, 1-11.

17. $ـ$ Solving three-dimensional layout optimization problems using fixed scale wavelets, Comput. Mech. 25 (2000), no. 2-3, 274-285.

18. A. R. Diaz, A wavelet-Galerkin scheme for analysis of large-scale problems on simple domains, International Journal for Numerical Methods in Engineering 44 (1999), no. 11, 1599-1616. MR 99m:80002

19. L. Escauriaza, E. B. Fabes, and G. Verchota, On a regularity theorem for weak solutions to transmission problems with internal Lipschitz boundaries, Proc. Amer. Math. Soc. 115 (1992), no. 4, 1069-1076. MR 92j:35020

20. Richard S. Falk and John E. Osborn, Remarks on mixed finite element methods for problems with rough coefficients, Math. Comp. 62 (1994), no. 205, 1-19. MR 94c:65136

21. P. Grisvard, Elliptic problems in nonsmooth domains, Pitman (Advanced Publishing Program), Boston, Mass., 1985. MR 86m:35044]

22. $ـ$ Singularities in boundary value problems, Masson, Paris, 1992. MR 93h:35004

23. N. Heuer and E. P. Stephan, The Poincaré-Steklov operator within countably normed spaces, Mathematical aspects of boundary element methods (Palaiseau, 1998), Chapman \& Hall/CRC, Boca Raton, FL, 2000, pp. 152-164. MR 2000j:65123

24. V. V. Jikov, S. M. Kozlov, and O. A. Oleŭnik, Homogenization of differential operators and integral functionals, Springer-Verlag, Berlin, 1994, Translated from the Russian by G. A. Yosifian [G. A. Iosifyan]. MR 96h:35003b 
25. R. Bruce Kellogg, On the Poisson equation with intersecting interfaces, Applicable Anal. 4 (1974/75), 101-129, Collection of articles dedicated to Nikolai Ivanovich Muskhelishvili. MR 52:14623

26. Carlos E. Kenig, Harmonic analysis techniques for second order elliptic boundary value problems, Published for the Conference Board of the Mathematical Sciences, Washington, DC, 1994. MR 96a:35040

27. A. V. Knyazev, Iterative solution of PDE with strongly varying coefficients: algebraic version, Iterative Methods in Linear Algebra (Amsterdam) (R. Beauwens and P. de Groen, eds.), Elsevier, 1992, Proceedings IMACS Symp. Iterative Methods in Linear Algebra, Brussels, 1991, pp. 85-89. CMP 92:11

28. Serge Levendorskii, Degenerate elliptic equations, Mathematics and its Applications, vol. 258, Kluwer Academic Publishers Group, Dordrecht, 1993. MR 95b:35079

29. J. L. Lions, Perturbations singuliéres dans les problémes aux limites et en contrôle optimal, Lecture Notes in Mathematics, vol. 323, Springer-Verlag, New York, 1973. MR 58:29078]

30. T.A. Manteuffel, S. McCormick, and G. Starke, First-order systems least-squares for secondorder elliptic problems with discontinuous coefficients, Proceedings of the Seventh Copper Mountain Conference on Multigrid Methods, Copper Mountain, CO, April 3-7, 1995, NASA Conference Publication 3339, Part 2, 1995, p. 551.

31. G. I. Marchuk, Yu. A. Kuznetsov, and A. M. Matsokin, Fictitious domain and domain decomposition methods, Soviet J. Numerical Analysis and Math. Modelling 1 (1986), 1-82.

32. William McLean, Strongly elliptic systems and boundary integral equations, Cambridge University Press, Cambridge, 2000. MR 2001a:35051

33. Serge Nicaise and Anna-Margarete Sändig, Transmission problems for the Laplace and elasticity operators: regularity and boundary integral formulation, Math. Models Methods Appl. Sci. 9 (1999), no. 6, 855-898. MR 2000i:35022

34. O. A. Oleinnik, A. S. Shamaev, and G. A. Yosifian, Mathematical problems in elasticity and homogenization, Studies in Mathematics and its Applications, vol. 26, North-Holland Publishing Co., Amsterdam, 1992. MR 93k:35025

35. R. Plato and Vainikko G., On the regularization of projection methods for solving ill-posed problems, Numerische Mathematik 57 (1990), 63-79. MR 91h:65097

36. W. Proskurowski and O. Widlund, A finite element - capacitance matrix method for the Neumann problem for Laplace's equation, SIAM Stat. and Sci. Comput. 1 (1980), 410-425.

37. Alfio Quarteroni and Alberto Valli, Domain decomposition methods for partial differential equations, Oxford University Press, Oxford, 1999. MR 83e:65164

38. Enrique Sánchez-Palencia, Nonhomogeneous media and vibration theory, Lecture Notes in Physics, vol. 127, Springer-Verlag, Berlin, 1980. MR 82j:35010

39. V. K. Saul'ev, On solving boundary value problems with high performance computers by a fictitious domain method, Siberian Math. J. 4 (1963), no. 4, 912, (In Russian).

40. Giuseppe Savaré, Regularity results for elliptic equations in Lipschitz domains, J. Funct. Anal. 152 (1998), no. 1, 176-201. MR 2000d:35046

41. V. V. Vasin and A. L. Ageev, Ill-posed problems with a priori information, Inverse and Illposed problems series, VSP, Utrecht, The Netherlands, 1995. MR 97j:65100

42. Olof B. Widlund, An extension theorem for finite element spaces with three applications, Numerical Techniques in Continuum Mechanics (Braunschweig/Wiesbaden) (Wolfgang Hackbusch and Kristian Witsch, eds.), Notes on Numerical Fluid Mechanics, v. 16, Friedr. Vieweg und Sohn, 1987, Proceedings of the Second GAMM-Seminar, Kiel, January, 1986, pp. 110-122.

Department of Mathematics, University of Colorado at Denver P.O. Box 173364,

Campus Box 170, Denver, Colorado 80217-3364

E-mail address: knyazev@na-net.ornl.gov

URL: http://www-math.cudenver.edu/〜aknyazev/

Courant Institute of Mathematical Sciences, 251 Mercer Street, New York, New YORK 10012

E-mail address: widlund@cs.nyu.edu

$U R L:$ http://cs.nyu.edu/cs/faculty/widlund/index.html 